\title{
The Fundamental Assumptions of the Theory of Relativity Shown False, Yet Many Predictions Match Observations. This Work Shows Why
}

\author{
Jacob Schaf \\ Universidade Federal do Rio Grande do Sul (UFRGS), Instituto de Fsica, \\ Porto Alegre, Brazil \\ Email: schaf@if.ufrgs.br
}

Received 21 August 2014; revised 16 September 2014; accepted 5 October 2014

Copyright (C) 2014 by author and Scientific Research Publishing Inc.

This work is licensed under the Creative Commons Attribution International License (CC BY). http://creativecommons.org/licenses/by/4.0/

(c) (i) Open Access

\section{Abstract}

The Theory of Relativity (TR) is now in conflict with a number of trustworthy experimental observations, most of which discovered recently with the help of the tightly synchronized clocks of the GPS. With base in these observations, the present work appoints mistaken assumptions in the construction of the TR, that beginned with a wrong interpretation of the null results of the Michelson light anisotropy experiments. The assumptions of the TR about the nature of space also become unfair within the scenario of the Higgs mechanism, underlying the Standard Elementary Particle Model, according to which space is filled up with real Higgs condensate (HC), responsible for giving mass to the elementary particles. The HC is a real and very powerful quantum space (QS), stable up to $\mathbf{1 0}^{15}$ degrees Kelvin that rules the inertial motion of matter and the propagation of light. This QS is the locally ultimate reference for rest and for motions of matter and propagates light at a well defined velocity $c$ with respect to the QS and not with respect to all inertial references. The presence of the HC cancels the reciprocal symmetry between observers that in the TR is the source of many unresolved or badly resolved paradoxes. It also eliminates the intrinsic isotropy of light with respect to all possible inertial references. On the other hand, the recent experimental observations show very clearly that this real QS is moving in the ordinary three-dimensional space according to a Keplerian velocity field round each astronomical body, consistently with the local main astronomical motions, thereby creating the observed gravitational dynamics. This spacedynamics is the quintessence of the gravitational fields and implies that earth is very closely resting with respect to the QS, which explains the null results of the Michelson light anisotropy experiments. All the conventional tests of the TR have been made with atoms or elementary particles at very high velocities within the earth based laboratories. In reality these experiments do not test the assumptions of the TR, but simply show the effect of motion with respect to the 
local QS. The equations of the TR describe well the corrections of time, mass, energy etc., however in these expressions the relative velocity must be replaced by the velocity with respect to the local QS. This spacedynamics has been shown in References [4]-[6] to correctly create the observed gravitational dynamics on earth and in the solar system as well as the galactic gravitational dynamics without the need of dark matter. This theory also correctly accounts for all the experimentally confirmed effects, caused by the gravitational fields on the propagation of light and the rate of clocks and moreover apoints the physical mechanism accelerating the expansion of the universe.

\section{Keywords}

\section{Relativity, Relativistic Effects, Space, Gravitational Physics, Gravitational Effects}

\section{Introduction}

The theory of relativity (TR) is a theory of space and time, of light and clocks. What is absolutely extraordinary is that Einstein has developed this theory in the age of Newtonian mechanics, of thermodynamics, of the steam engines and trains. In this epoch the measuring techniques were rather limited and the experimental survey was poor. Years before, Maxwell had published his top work about electrodynamics, in which he imagined the electromagnetic waves propagating in a static ether that however entails unusual physical properties. In the epoch Michelson just had obtained very precise measurements of the velocity of light. Also the gravitational dynamics of the solar system was rather well understood in terms of Newton's universal gravitation. Moreover, the motion of the solar system with respect to nearby stellar constellations too was known. Throughout progress in science was going well. However, then the null results of the Michelson light anisotropy experiments have surprised the scientific community and spread out confusion. How could light be isotropic with respect to earth if earth is moving round the sun at $30 \mathrm{~km} / \mathrm{sec}$ and even at much higher velocity with respect to other astronomical objects?

In his university studies Einstein had learned about the Galilean principle of relativity, according to which local experiments of mechanics cannot reveal the sate of uniform motion of the laboratory along a straight line and hence the laws of mechanics work equally well in all the possible inertial references. When Michelson announced the null results of his light anisotropy experiments, many scientists began searching for genuine physical causes. Nevertheless, Einstein, an outsider in the epoch and thus less constrained by conventional views, tried to understand these null results putting in doubt the concepts of absolute space and absolute time and rejecting the idea of the static ether. In Einstein's view, the null results of the Michelson light anisotropy experiments demonstrated that local electromagnetic experiments too cannot reveal the state of motion of the laboratory. This was the starting motivation of the now well known TR.

The ground laying assumptions of the TR [1] are that empty space in itself contains nothing objectively real that can represent a reference for motions. This means that no absolute references can be defined and that the idea of absolute motions has no meaning. On the other hand however, the idea that matter particles have mass and are objectively real in themselves remained intact. In this view the elementary particles interact by various force fields, generating the observed dynamics in the material world. Moreover, the fact that light can propagate in empty space, was seen as prove that light needs no physical medium to propagate (no ether). In addition, the nominally null result of the Michelson light anisotropy experiments was interpreted as evidence that the velocity of light is intrinsically constant and isotropic with respect to all possible inertial references.

According to the TR, only relative motions between matter bodies are relevant in physics. The inevitable conclusion of this is that all the possible inertial references (IRs) are equivalent and that the laws of physics, discovered in one given IR, must be good in all the other possible IRs. This is known as the principle of relativity or covariance of the laws of physics. Accordingly the form of Maxwell's equations must have the same form (Lorentz invariance) in all the IRs. As these equations give the velocity of light, the velocity of light must be the same when measured with respect to all possible IRs, independently from the velocity of the sources or of these IRs. Einstein promoted this constancy and intrinsic isotropy of light to the status of a fundamental law of physics. It however is important to note here that, in Einstein's view, the velocity of light is the one measured by the light go-return roundtrip along a known distance and clock method. In Section 4 of the present work, it will be shown 
that this measuring method is constrained to give always the same result, independently from the velocity of the laboratory with respect to the real Higgs quantum space ruling the inertial motion of matter and the propagation of light, because the light roundtrip time and the oscillation roundtrip time (period) of the time standard, by which the clock counts time, are affected by the velocity of the laboratory with respect to the Higgs quantum space in exactly the same proportion, as evidenced by the Ives-Stilwell experiment [2]. Hence, this measuring method is misleading and the velocity of light measured in this way is an experimental artifact.

There is no need to reproduce here the steps by which Einstein constructed the TR. The details can be found in Einstein's original publications, or in translated collections like, for instance that in Ref. [1]. They also are carefully described in many good textbooks [3]. Einstein's development of the TR will be discussed in detail in Section 4 of the present work, in the light of recent new experimental observations and theoretical developments. The present work does not follow a chronological sequence, but is developed according to a simple logic: The more fundamental aspects precede the less fundamental ones. This is essential in order to avoid unfounded assumptions. In the construction of the TR, Einstein did not follow this order. He interpreted the null results of the Michelson light anisotropy experiments in the scenario of the Newtonian celestial mechanics, without knowing the true kinematical circumstances of the non inertial earth based laboratories. Obviously this non-inertial character entails implicit kinematical circumstances that Einstein totally ignored in the epoch. Therefore, his interpretation of the null results of the light anisotropy experiments was fated to be incorrect.

The experiments on the velocity and anisotropy of light, underlying the TR, as well as the experiments supposedly testing the predictions of the TR were performed within the non-inertial earth based laboratories that are accelerated upward, due to implicit kinematical circumstances created by the earth's gravitational field. Hence, in order to make a meaningful discussion of the light anisotropy experiments and of the fundamental assumptions of the TR, it is absolutely essential to unveil first the exact kinematical circumstances of the earth based laboratories. This becomes possible only knowing the exact nature of space and its role in the gravitational physics. Otherwise such a discussion will go in circles and lead to mistaken views. Therefore, it is absolutely necessary to anticipate considerations about the nature of space and matter before attempting any interpretation of the Michelson light anisotropy experiments as well as of other observations. In the forthcoming Section 2, the nature of space and matter will be discussed in the light of the Higgs mechanism of the Standard Elementary Particle Model (SEPM) that can provide us with a reliable view about the nature of space and its role in the gravitational dynamics. The Higgs condensate (HC) is a very powerful quantum space (QS), responsible for giving mass to the elementary particles by the Higgs mechanism and hence rules the inertial motion of matter and of energy (propagation of light). Moreover, in Section 3 the conclusions enforced by the recent experimental observations, achieved with the help of the tightly synchronized atomic clocks in orbit (GPS) will be outlined.

It can be advanced here that all these new experimental facts, as well as the presence of the HC, frontally contradict the fundamental assumptions of the TR. Nevertheless, in Section 4 it will be shown that, despite the wrong assumptions in the TR, many of its predictions apparently match the experimental observations, within the circumstances and the precision they effectively have been tested. In all of these circumstances, the predictions of the TR are indistinguishable similar to those predicted by the theory of spacedynamics [4]-[6]. The reason is simple. All the effectively realized experiments, that are claimed to confirm the predictions of the TR, have been made within earth based laboratories. According to recent experimental observations to be described in Section 3, the earth based laboratories truly rest very closely with respect to the QS (HC), ruling the propagation of light and the inertial motion of matter, in the Keplerian velocity field of the QS, creating the earth's gravitational field, the solar and galactic gravitational fields. Hence, in fact these experiments do not test the TR, but simply are the effects of motion with respect to the QS, that is, motion with respect to the earth based laboratories that are closely commoving with the QS in the solar and galactic velocity fields. In conclusion, despite the fundamental assumptions of the TR are false, its predictions, within the extend they effectively have been tested, seem to match the experimental observations. What is wrong is generalizing them to all the other non tested experimental circumstances and imputing them to relative velocity. Hence, in the view of the present work, in the expressions, found by Einstein for time dilation, for mass and for energy, the relative velocity with respect to Einstein's hypothetical inertial references must be replaced by the velocity with respect to the QS.

\section{The Higgs Condensate as a Real Quantum Space, Ruling the Inertial Motion of Matter and the Propagation of Light}

According to the SEPM, space in filled up with the Higgs field, a Bose-Einstein (BE) condensate of the zero 
spin Higgs bosons. The Higgs condensate (HC) is a very powerful quantum space (QS) stable up to $10^{15}$ degrees Kelvin. A more detailed description of the BE condensates, specifically of the superconducting condensate and the Higgs condensate as well as their physical phenomenologies, relevant to the present work, has been given by the author in References [4]-[6]. Here only a succinct outline will be given.

In the gauge theories, all the elementary particles are originally massless and move at the velocity of light. Nature however tells a different story. With only a few exceptions, all the elementary particles have inertial mass. The well known theory of superconductivity, in which the $\mathrm{U}(1)$ gauge symmetry is spontaneously broken during Bose-Einstein condensation of the Cooper electron pairs, fixing a gauge and the fact that superconductors confine the electromagnetic field by the Meissner effect, making it short-range and giving mass to the photons within superconductors, was an important hint to solve the problem of mass acquisition by the elementary particles [7]. In superconductors BE condensation of the Cooper electron pairs occurs at a low critical temperature, due to the quantum phase correlation (BE correlation) between the wave functions of the bosonic particles. This correlation entangles the particles, making them indistinguishable, breaking the U(1) gauge symmetry and enforcing long-range phase coherence. The BE phase coherent superconducting condensate is described by a complex order parameter $\psi=\psi(r) \mathrm{e}^{i \theta}$. During the BE condensation the BE correlation creates a negative potential energy term (bonding term) that is proportional to the boson density $\rho=\psi^{*} \psi$. In addition an interaction term (anti-bonding term) proportional to $\left(\psi^{*} \psi\right)^{2}$ takes place. However, the coefficient of the bonding term is considerably larger than that of the anti-bonding term. Therefore within the effective negative potential well in the form of the Mexican sombrero, the boson system can lower its energy by condensing into a state of long-range phase coherence assuming a well defined phase between zero and $2 \pi$. To the minimum of energy in the Mexican sombrero potential corresponds a well defined volumetric density $\rho=\psi^{*} \psi$, whose value depends on the $\mathrm{BE}$ correlation. The volume of the condensate cannot collapse because of the interaction term. If the density exceeds the equilibrium value, the system will climb up in the external wall of the potential well. In this case, the condensate can lower its energy by expanding the volume.

According to Quantum Field Theory (QFT), underlying the Standard Elementary Particle Model (SEPM), the zero spin Higgs bosons too undergo BE condensation, spontaneously breaking the U(1) gauge symmetry and forming a phase coherent Higgs Condensate (HC) [8]-[10]. In the view of the QFT of the SEPM, space is filled up with the Higgs condensate (HC), a very powerful Quantum Space (QS), stable up to $10^{15}$ degrees Kelvin [7]. If actually the BE condensation of Higgs bosons still is happening in the universe, it may be more intense in colder regions between the galaxies. If the volumetric density $\rho=\psi^{*} \psi$ of the $\mathrm{HC}$ is beyond the equilibrium density, the energy state of the universe is not minimum. It may lower its energy by volumetric expansion, thereby displacing the energy state toward the minimum, especially in the colder regions between galaxies. Such continuing BE condensation can be the physical mechanism that drives the accelerated expansion of the universe.

The HC plays in space an analogous role as the superconducting condensate (SCC) plays in superconductors. Likewise the SCC confines the electromagnetic field down to the order of hundreds of nanometers by the Meissner effect, the HC confines the matter fields (weak and strong nuclear fields) down to about $10^{-19} \mathrm{~m}$, making them short-range, providing the elementary particles (hadrons and leptons) with inertial mass and hence with mechanical properties by the Higgs mechanism [8]-[10]. This will say that the HC is a QS that rules the inertial motion of matter and the propagation of light and hence is the ultimate (locally absolute) reference for rest and for motion of matter and light.

BE condensates couple only to very specific fields. The SCC is well known to couple to electromagnetic (EM) fields. Electric and magnetic fields cause local phase displacements of the superconducting order parameter with respect to the overall phase. Such phase displacements involve energy, because they must conquer with the phase correlation, climbing up in the potential energy. Phase displacements represent phase disorder, elevating the energy of the system and tending to recover the U(1) gauge symmetry. The SCC can lower its energy by confining or expelling the magnetic field, which is the Meissner effect. It is important to realize that local phase displacements are necessarily associated with local motion of the condensate (currents). A constant phase gradient entails uniform motion of the condensate and phase changing with time corresponds to accelerated motion. In superconductors these currents generate the Lorentz reaction force field that is the drive of the Meissner effect. Motion of the SCC along closed loops, once excited, automatically becomes persistent.

The atoms of the SC material do not perceive the presence of the SCC itself, because there is no interaction, no scattering. They however perceive the vector potential, due to its motion and acceleration. Analogously the 
presence of specifically the HC is imperceptible to us. We however perceive the gravitational field that it generates on moving through us according to a non-uniform velocity field. This is not strange. We even do not observe the atmosphere itself, however perceive easily its perturbations (sounds, wind).

The phenomenologies in the HC are totally analogous to those in the SCC. The constitution of the HC is different from the SCC. It does not couple to EM fields nor to gravitational fields. It couples specifically to weak and strong nuclear fields. However, displacing the phase of the Higgs order parameter certainly involves much-much higher energy than in the SCC. The weak and strong nuclear fields cause phase disorder in the Higgs order parameter, elevating its energy. As the HC permeates all of space, it can minimize its energy only by confining the weak and strong nuclear fields, which is the Higgs mechanism that gives mass to the elementary particles porting weak hypercharge.

Cyclic excitations in the SCC as well as in the HC are intrinsically quantized and are persistent due to the phase correlation and perfect conservativeness of the condensates. This links the inertial motion of the elementary particles directly to the persistence of the excitations in the HC. On the other hand acceleration of the elementary particles with respect to the HC is associated with additional phase distortions that too must conquer with the phase correlation of the HC. This additional phase distortion costs additional energy (producing additional mass) and too becomes persistent. From this viewpoint, inertial mass and the conservation of the (relativistic) linear and angular momentum of matter bodies is due to the persistence of the excitations in the HC.

The Higgs mechanism likewise the Meissner effect in superconductors certainly entails macroscopic phenomenologies. Superconductors minimize energy developing macroscopic screening currents and a macroscopic Lorentz reaction force field, expelling the magnetic field out from the superconductor or compressing it into regions where the superconducting order parameter is weaker or absent. The HC likewise the SCC minimizes its energy developing a macroscopic screening velocity field of the HC. As the HC rules the inertial motion of matter, such screening velocity fields, if non-uniform, create an inertial dynamics, that is, gravitational effects, macroscopically thrusting the matter fields along the matter density gradient toward regions where the Higgs order parameter has already been depressed by large amounts of matter. The macroscopic screening velocity field of the Higgs condensate round matter bodies is to be seen as the Keplerian velocity field of the QS in the theory of spacedynamics, responsible for the gravitational fields and the corresponding force field is the gravitational field that in fact is a centrifugal (fictitious) force field toward the gravitational center. Please see References [4]-[6] for details.

\section{The Nature of Space and of the Gravitational Physics in the Light of the Recent New Experimental Observations}

According to the TR empty space is the absolute nothingness. It contains nothing that can represent a reference for motions. Very contrarily, according to recent developments in the SEPM, space is filled up with the Higgs condensate, a very powerful quantum space (QS), ruling the inertial motion of the matter particles and the propagation of light. However, is this QS static or is it dynamic? Recent new experimental observations, achieved with the help of the tightly synchronized clocks of the GPS and to be described hereafter, show very clearly that the real QS, is moving round earth, round the sun and round the galactic center consistently with the local main astronomical motions. In this spacedynamics the earthglobe (center of earth) and the other planets of the solar system, as well as astronomical bodies in general throughout the universe are very nearly resting with respect to the local moving real QS. Please see Section 3.2 for details. Light is very nearly isotropic with respect to all these bodies, not because of the intrinsic isotropy postulated in the TR, however because earth and astronomical bodies in general very closely rest with respect to the QS. This straightforwardly explains the null results of the Michelson light anisotropy experiments. Note that this spacedynamics has nothing to do with the ether drag. The Keplerian velocity field of the QS, which is the quintessence of the gravitational fields, is shown in Refs [4]-[6] to correctly create the observed gravitational dynamics on earth and in the solar system. It also accounts for the gravitational dynamics of galaxies without the need of dark matter and predicts the accelerated expansion of the universe. It moreover straightforwardly explains all the observed effects, caused by the gravitational fields on the propagation of light and the rate of clocks.

\subsection{The One-Way Anisotropy of Light}

Clocks are mechanical or electronic devises that count time, using a stable time standard. A time standard is the 
period of a mechanical or quantum oscillator. The precision of the clock depends on the stability of the time standard. Actually the best time standards, used in the precise atomic clocks, are the very stable periods of electromagnetic (EM) oscillations due to the hyperfine transition of excited Cs or Rb atoms. These EM oscillations are go-return roundtrips of the electromagnetic field that are affected by motion of the laboratory in exactly the same proportion as light go-return roundtrips, as shown in the Ives-Stillwell experiment [2]. Therefore measuring the velocity of light or distances by the usual method of light go-return roundtrips and clock are fated to give always the same result regardless the velocity of the laboratory. They are experimental artifacts.

Using Einstein's synchronization method, the very stable atomic clocks of the GPS can be collectively synchronized altogether to within 0.1 ns (time for light to travel $3 \mathrm{~cm}$ ). For low orbit satellites, clock synchronization can even be up to an order of magnitude better. With the help of these tightly synchronized atomic clocks, the one-way travel time of electromagnetic (EM) signals (light) between these satellites has been precisely measured. Especially precise and clear-cut measurements of the one-way velocity of EM signals (light) was achieved using the twin satellites of the Gravity Recovery and Climate Experiment (GRACE).

The twin satellites of GRACE project move in the same sense at nearly $8 \mathrm{~km} / \mathrm{sec}$ along coplanar and practically identical circular polar orbits at $500 \mathrm{~km}$ of altitude, separated from each other by $200 \mathrm{~km}$ and their positions being monitored by the GPS within $3 \mathrm{~cm}$. To measure microgravity effects, these satellites need to be equipped with highly stable atomic clocks, synchronized to better than 0.16 ns. The satellites continuously exchange EM signals between them in both senses. It is observed that the signal transit time from the leading satellite to the rear satellite corresponds to a shortening with respect to the expectation from the TR by more than $5 \mathrm{~m}$ (17 ns), while the signal transit time from the rear satellite to the leading satellite is lengthened by more than $5 \mathrm{~m}$ (17 ns) [11]. These observations are consistent with backward signal anisotropy of nearly $8 \mathrm{~km} / \mathrm{sec}$ with respect to the satellites, which is exactly the velocity of the satellites.

This one-way anisotropy of EM signals (light) shows unambiguously that the EM signals take a longer time from the rear satellite to the leading satellite and shorter time from the leading satellite to the rear satellite by exactly the same value. As the GRACE satellites form an inertial reference common to both satellites, this fact unquestionably contradicts the fundamental assumption of the TR, according to which the signal should take the same time in both senses. This experiment proves that a real spatial medium (QS) exists that propagates light at a characteristic constant velocity $c$ and that this QS does not move within the earth's non-rotating reference along a North-South direction. Hence, this anisotropy is due uniquely to the motion of the satellites.

The immediate consequence of this experimental observation is the absolute need of revising the interpretation of all the Michelson light anisotropy experiments, made within earth based laboratories during the past century. Most of the Michelson experiments have measured the light anisotropy due to the orbital and cosmic motion of earth. Systematically all these experiments obtained nominally null results. Now this must be interpreted as proving that the earthglobe has no relevant velocity with respect to the real QS that rules the propagation of light. Obviously this can make a sense only if this QS is moving with earth round the sun and with the solar system round the galactic center etc. This conclusion may seem reckless. However, it has been demonstrated (see Refs. [4]-[6] to be exactly the spacedynamics that correctly creates the observed gravitational dynamics.

Other light anisotropy experiments, using highly precise Michelson interferometers, intended to measure the anisotropy of light with respect to the earth based laboratory itself. They obtained anisotropy of about $8 \mathrm{~km} / \mathrm{sec}$, constant the whole day and the whole year [12]. This anisotropy gives evidence that real QS, ruling the propagation of light, is moving through the earth based laboratories and round earth at nearly $8 \mathrm{~km} / \mathrm{sec}$ on surface, not along a North-South direction, however along West-East direction, in the sense of the Moons orbital motion. This corresponds exactly to the Keplerian velocity field of real QS creating the gravitational dynamics on earth. Please see Refs. [4]-[6] for details.

\subsection{Absence of the Gravitational Time Dilation of the Solar Gravitational Potential on the GPS Clocks}

An even much stronger and persuasive prove, showing that real QS, ruling the inertial motion of matter and the propagation of light, is moving round earth and round the sun according to a velocity field consistent with the local main astronomical motions, is provided by the absence of gravitational time dilation of the solar gravitational potential on the GPS clocks, which is predicted by General Relativity (GR) but not observed. This is the 
well known noon-midnight problem [13] [14]. During the orbital motion of earth round the sun, the six orbital planes of the 24 GPS satellites alternate them in the plane nearly parallel to the earth-sun axis, passing 6 hours closer than earth from the sun and then 6 hours farther from the sun.

The effect of the solar gravitational potential $(U)$ on the rate of the GPS clocks, predicted by GR, is given by $T(R)=T_{0}\left[1-2 U / c^{2}\right]^{-1 / 2}$ where $T_{0}$ is the time period in the absence of a gravitational potential and $c$ is the velocity of light. To first approximation this time dilation effect is proportional to $U / c^{2}$. Such gravitational time dilation effects on the atomic clocks within the earth's gravitational field have been well observed and corresponding time dilation effects certainly would be shown by clocks fixed within the solar gravitational field too. According to GR the delay, accumulated during the 6 hours of closest approach from the sun, is larger than 24 ns, which would be recovered during the subsequent 6 hours farthest from the sun. The resulting 12 hour periodic sinusoidal deviation in the time display of these clocks would be more than two orders of magnitude larger than the stability of the clocks and, if present, would be very easily observed. Nevertheless, contradicting the prediction of GR, no sign of such variation is observed [13] [14]. Note that this gravitational time dilation, predicted by GR, cannot be canceled by the special relativistic time dilation, due to changes of velocity of the satellites with respect to the solar non-rotating reference, because this would be two orders of magnitude larger than the one predicted by GR and too is not observed. Observations show that the rate of the terrestrial and GPS clocks is ruled exclusively by the earth's gravitational field. This is fortunate, because otherwise the use of the GPS would be a lot more complicated. GR cannot explain the absence of this time dilation because the gravitational potential is a scalar that depends on the distance from the gravitational center but not on the orbital velocity of earth.

Time dilation is well known to be caused by motion, as evidenced by the well known increased lifetimes of speeding Muons and the redshifts of the radiation emitted by speeding Hydrogen atoms [2]. In the STR this time dilation effect is imputed to the relative velocity $\left(v_{r}\right)$ with respect to the laboratory observer. To first approximation this effect is proportional to $\left(v_{r} / c\right)^{2}$. Obviously the gravitational slowing of the atomic clocks within the earth based laboratories cannot be due to relative velocity because these clocks rest with respect to the laboratory observer. However, if motions cause time dilation, why then does the orbital motion of earth suppress the time dilation caused by the solar gravitational field on the earth based and GPS clocks? Absurdly in one case motion causes time dilation and in the other case it suppresses it. This contradiction lets clear that time dilation is not caused by gravitational potential. On the other hand, the slowing of the atomic clocks in the earth based laboratories cannot be caused by relative velocity. Hence, time dilation necessarily is caused by an implicit velocity of a more fundamental nature, which will be seen to be the velocity with respect to QS (Higgs condensate).

In the beginning of the previous Subsection 3.1 it was asserted and the Ives-Stilwell experiments prove that the time standards, by which clocks count time, are slowed by motion with respect to the QS in the same proportion as light roundtrips. Therefore, it seems obvious that the observed gravitational slowing of the atomic clocks on earth is related with the observed small constant anisotropy of light of nearly $8 \mathrm{~km} / \mathrm{sec}$. Both these effects are proportional to $v^{2} / c^{2}=8^{2} / c^{2}$ ( $c$ in $\mathrm{km} / \mathrm{sec}$ ). Similarly the absence of effects due to the solar gravitational potential on the GPS clocks must be related with the well known absence of light anisotropy due to the orbital motion of earth. The mysterious implicit velocity $\left(V_{i m}\right)$ of the earth based laboratories that causes the small light anisotropy of nearly $8 \mathrm{~km} / \mathrm{sec}$ is the same that causes the well observed small decrease of the clock rates on earth. On the other hand, the orbital motion of earth $(30 \mathrm{~km} / \mathrm{sec})$ that suppresses the gravitational time dilation due to the solar gravitational field, also suppresses the light anisotropy, caused by the implicit velocity $\left(V_{\text {im }}\right)$ in the solar gravitational field. This singles out velocity with respect to the real QS as the unified cause of time dilation. It is the usual velocity with respect to the earth based laboratories (note that earth is very nearly resting with respect to the QS propagating light) and it is this mysterious implicit velocity $V_{\text {im }}$ in the case of clocks fixed within gravitational fields.

The only possible way to provide physical reality to this implicit velocity $V_{i m}$ is acknowledging the existence of a real spatial medium (Higgs condensate or QS) that rules the inertial motion of matter and the propagation of light and hence is the ultimate (locally absolute) reference for rest and for motions of matter and light. According to references [4]-[6] this QS is moving in the ordinary three dimensions round earth according to a velocity field (at $8 \mathrm{~km} / \mathrm{sec}$ on surface) consistently with the Moon's orbital motion, as well as round the sun according to a velocity field consistent with the earth's orbital motion (30 km/sec).

Obviously earth cannot be kinematically privileged in detriment to all the other planets of the solar system 
and astronomical bodies in general throughout the universe. Earth is not the only planet commoving with the real QS in the velocity field round the sun. All the planets must be closely commoving with the real QS. This will say that the real QS is moving according to a Keplerian velocity field round the sun:

$$
\boldsymbol{V}(r)=[\gamma M / k]^{1 / 2} \boldsymbol{e}_{\phi}
$$

where $\gamma$ is the gravitational constant, $r$ is the radial spherical coordinate and $\boldsymbol{e}_{\phi}$ is the unit vector along the azimuthal spherical coordinate. This Keplerian velocity field is the quintessence of the gravitational fields and is responsible for the gravitational dynamics, observed within the gravitational fields. The velocity field Equation (1) has been shown in References [4]-[6] to appropriately create the gravitational field of earth and of the sun and to naturally explain all the effectively observed effects of the gravitational fields on the propagation of light and on the rate of the clocks. It also fully corroborates the propositions made in Section 3.1 in relation to the null results of the Michelson light anisotropy experiments. Such a Keplerian velocity field of real QS must be circulating round each matter concentration throughout the universe, generating the respective gravitational fields.

The disk shape of the solar system and of the galaxies as well as of the satellites and planetary rings round planets all show that the orbits of the natural astronomical bodies are closely concentrated about the equatorial plane of the respective Keplerian velocity field, which minimizes their velocity with respect to the real QS. This will say that the astronomical bodies in general very closely rest with respect to the moving real QS, which explains why the Michelson experiments gave null results. The circular orbital motions of the planets need not to be explained anymore because it is QS itself that so moves. The very slow implicit velocity of the planets of the solar system of only about a hundred of $\mathrm{m} / \mathrm{sec}$ is ruled by the principle of inertia. This very low velocity gives rise to the very small deviations from the circular equatorial orbits. The vertical free fall is an extreme case of elliptic orbits in which the opposite implicit velocity of the body with respect to real space just compensates the Keplerian velocity of the QS.

The experimental evidence that the QS, ruling the inertial motion of matter and the propagation of light, is circulating round earth and round the sun according to a Keplerian velocity field certainly is a very significant result. However, what is really important and auspicious in this result is that this Keplerian velocity field of the QS is precisely the one that correctly creates the observed gravitational dynamics on earth, in the solar system as shown in References [4]-[6]. There it also has been shown to correctly generate the galactic gravitational dynamics without the need of dark matter and to correctly generate in terms of well known and genuine physical effects all the effectively observed effects of the gravitational fields on the propagation of light and on the rate of clocks.

The only relevant velocity of the earth based laboratories with respect to the real QS is due to the local velocity field creating the gravitational field of earth itself. The effect of this velocity is proportional to $\left(v^{2} / c^{2}\right)$, which is in the order of only $10^{-10}$. Excepting some Michelson light anisotropy experiments and some Mössbauer experiments, the sensitivity of all the other so called relativistic experiments is much too low to evidence such small effects. The velocities in all of the so called relativistic experiments are many orders of magnitude larger than the $7.5 \mathrm{~km} / \mathrm{sec}$ of the earth based laboratories with respect to the real QS (hydrogen atoms in the IvesStillwell experiment $\left(v \sim 2 \times 10^{3} \mathrm{~km} / \mathrm{sec}\right)$ [2], the increased lifetimes of Muons in cosmic rays $(v \sim c)$, the relativistic mass of electrons and protons from particle accelerators $(v \sim c)$ or of the relativistic energy term in atomic and nuclear states). Only very recently have the measuring techniques achieved sensitivity enough to put in evidence the very low effects due to the actual velocities of the earth based laboratories with respect to real QS, in which the atomic clocks have played a central and fundamental role.

\subsection{Non-Synchronous Arrival of the Millisecond Pulsar Signals to Equidistant Earth Based Antennas}

The resolution of images from very distant astronomical objects can be improved by orders of magnitude by interferometric methods. The condition is that reception of the signals by the earth based telescopes or antennas are synchronous. This has been achieved to within 0.1 ns with the help of the GPS clocks. Digital image signals, recorded in the distant observatories, can be coherently mixed together with the help of computers, generating the improved images. According to the theory of relativity (TR), arrival of EM signals to the earth based antennas, equidistant from the source, should be synchronous. On testing the synchrony of the atomic clocks in the set ups for Very Long Baseline Interferometric (VLBI) radio-astronomy observations by the arrival of the wave 
fronts from distant millisecond pulsar signals, a surprise. While for antenna arrays along directions transverse to the earth's orbital motion the arrival is synchronous, for arrays along the orbital motion of earth they reach the foregoing antenna up to $4.2 \mu$ s before the rear antenna [13] [14]. This relatively enormous discrepancy exceeds the time resolution of the experiment by more than three orders of magnitude.

The aberration of stars cannot explain this non-synchronous arrival. It necessarily involves genuine refraction of the pulsar wave fronts in the environment of the solar system. In References [4]-[6] it is shown that the same refraction mechanism involved in the spacedynamic gravitational mechanism also gives rise to the gravitational deflection of light (the gravitational light lensing effect) and to the non-synchronous arrival of the millisecond pulsar wave fronts to the equidistant earth based antenna arrays along the orbital motion of earth, while letting the arrival synchronous to antenna arrays along directions transverse to the earths orbital motion. This is a spectacular confirmation of the spacedynamic gravitational mechanism.

\section{The Fundamental Assumptions of the Theory of Relativity in the Light of the Higgs Quantum Space Dynamics}

The present work will show that the predictions of the Theory of Relativity (TR) only apparently match the experimental observations. In fact all the actually performed experimental tests of the TR have been made for motions with respect to the earth based laboratories. However, recent experimental observations, described in Section 3, prove that the earth based laboratories are very nearly stationary with respect to the Higgs-QS, ruling the inertial motion of matter and the propagation of light, which is moving round the sun and round the galactic center according to a Keplerian velocity field, consistent with the local main astronomical motions, thereby generating the observed gravitational dynamics. This lets clear that, while the assumptions of the TR are totally general, the experimental tests have been made only within very specific circumstances. To make general conclusions from these observations clearly is unfair. Moreover, the results of these experiments, testing the predictions of the TR, are ambiguous because high velocities with respect to the earth based laboratories are almost equally high velocities with respect to the QS. Within their resolution these experiments cannot distinguish between the effects, due to relative velocity from those due to velocity with respect to the QS.

In the coming sub-sections it will be shown why, despite the fundamental assumptions of the TR about the nature of space and the velocity of light are false, many predictions of the TR seem to match the experimental observations within the true kinematical circumstances in which these experimental tests effectively have been made. In all of his thought experiments, Einstein assumed that one observer is hypothetically resting and another one is moving. The hypothetical kinematical circumstances of the resting observer in fact accomplish those of the earth based laboratories in which the experimental tests effectively have been carried out. Hence, the expressions, describing the so called relativistic effects, obtained by Einstein, in fact describe the effects due to motions with respect to the QS. The only necessary change in these expressions is replacing the relative velocity by the velocity with respect to the QS.

\subsection{The Fundamental Assumptions of the TR about the Nature of Space Are False}

The ground laying assumptions of the Theory of Relativity (TR) are that empty space in itself contains nothing objectively real that can represent a reference for motions of matter particles, which are seen as real in themselves [1]. This means that no absolute references can be defined and that the idea of absolute motions is meaningless. Hence, only relative motions between matter bodies and their interactions are relevant in physics. Moreover, the fact that light propagates in empty space has lead to the conclusion that light needs no physical medium to propagate. Moreover the nominally null results of the Michelson light anisotropy experiments were interpreted as evidence that the velocity of light is intrinsically constant and isotropic within all possible inertial references.

In Einstein's epoch the measuring techniques were rather limited and the experimental survey was poor. With the vertiginous development of the scientific research technologies along the last hundred of years, especially those related with fast electronics and the atomic clocks in orbit, the amount and quality of experimental results has enormously improved. Moreover, the great development of theoretical physics, especially of the quantum theory, has changed a lot our view about fundamental physics, about the physics of elementary particles, about the nature of empty space, about astrophysics and about the universe. However, in spite of all this progress, our understanding of the gravitational physics and of the gravitational fields has gone in circles. One reason is that 
Einstein's gravitational theory has made such a huge advance in this field that new improvements seemed unnecessary or even impossible. However, the main reason seems to be the fact that our physics knowledge in the microscopic world, despite the tremendous advance during the last century, is rather split up from the physics in the macroscopic world. GR is incompatible with the quantum theory. Quantum physics is impotent in the gravitational physics. It certainly is not obvious how to fit together the physics of the small with the physics of the large. One of the major obstacles certainly is the mistaken view about the ontological nature of space and of the material world. While in quantum physics the players are the interacting elementary particles, seen as real in themselves, in the gravitational physics the player is the intangible and endless space, seen as the absolute emptiness, a completely different protagonist. Visibly the prerequisite-number-one to find a solution to this impasse is uncovering first the true nature of space and its true connection with the elementary particle world. Actually the Quantum Field Theory (QFT) underlying the Standard Elementary Particle Model (SEPM) is proposing such a real space and its connection with the elementary particles.

In opposition to the nothingness of empty space in the TR, the SEPM entails the idea that space is filled up with the Higgs Condensate (HC), responsible for giving mass to the elementary particles. Although some nostalgic physicists see the HC as a quantum ether, the HC is a macroscopic quantum state, a completely different ontological entity. It is a Bose Einstein (BE) condensate of the zero spin Higgs bosons, a quantum fluid in which the Higgs bosons are completely entangled and indistinguishable. However, despite no parts can be distinguished in quantum fluids, they entail very stable excitations, that are intrisically quantized dynamical states (known as quasi-particles in superfluids and superconducting condensates). The HC is totally analogous to the superconducting condensate. However, while the Cooper pairs condense only at very low temperatures, the HC condenses at $10^{15}$ degrees Kelvin and is stable up to such high temperatures. This means that the BE correlation between the Higgs bosons is very strong. The HC is a very powerful and objectively real Quantum Space (QS), ruling the inertial motion of matter and the propagaton of light. Analogously as the superconducting condensate confines the electromagnetic field and provides inertial mass to the photons within superconductors, the HC confines the weak and strong nuclear fields, providing mass and hence mechanical properties to the elementary particles (please see Section 2 of the present work and Reference [5] [6] for more details).

The HC is not only a reference for the motions of matter and light, but effectively rules these motions and hence is locally their ultimate reference for rest and for motions. By introducing the Higgs-QS, the SEPM somehow has restored the objective reality of the physical space, which has been refuted in the TR. In a certain form the ontological hierarchy between matter and space, presumed in the TR, is being inverted. While the QS is objectively real in itself, the matter particles are simply like local quantized excitations, propagating in this real QS. On the other hand, the recent new experimental observations, achieved with the help of the tightly synchronized clocks of the GPS (see Section 3), show very clearly that this QS, ruling the inertial motion of matter and the propagation of light, is not static, but is circulating likewise a fluid round earth, round the sun and certainly round all the matter bodies throughout the universe according to a Keplerian velocity field (Equation (1)), consistent with the local main astronomical motions. This Keplerian velocity field of the QS is the quintessence of the gravitational fields and the observed astronomical motions throughout the universe closely trail the local motions of the QS. In Refs. [4]-[6] it is shown that this Keplerian velocity field straightforwardly and correctly creates the gravitational dynamics on earth, in the solar system and also can correctly generate the observed gravitational dynamics within galaxies without the need of dark matter. In this spacedynamics earth is very closely resting with respect to the local QS, which directly predicts the null results of the Michelson light anisotropy experiments.

The recent new experimental facts, described in Section 3, all frontally contradict the assumptions of the TR about the nature of space and the intrinsic isotropy and constancy of the velocity of light. They demonstrate that the (one-way) velocity of light is constant and isotropic not with respect to all possible inertial references, however with respect to the local moving QS, with respect to which earth and the other planets of the solar system, as well as the solar system and astronomical bodies in general throughout the universe are very nearly resting. Celestial mechanics basically is spacedynamics. The velocity of light is very nearly isotropic with respect to all these bodies, not because of the intrinsic isotropy of light, however because of the closely similar kinematical circumstances of all these bodies with respect to the QS. Within their precision the conventional experiments, testing the predictions of the TR, cannot distinguish between the effects due to the velocity with respect to the QS and the velocity with respect to the earth based laboratories, because, for high velocity, these effects have practically the same magnitude. Moreover, the new experimental observations in Section 3.2 also show very 
clearly that the rate of clocks is neither ruled by relative velocity nor by the gravitational potential, however by velocity with respect to the QS. Therefore the GPS clocks, moving with earth round the sun and hence nearly resting with respect to the QS in the solar Keplerian velocity field, do not show the effects of the solar gravitational potential, predicted by GR. According to GR, the GPS clocks should be delayed with respect to clocks on ground up to about 24 ns during the 6 hours in the part of their orbit closer than earth from the sun, which would be recovered in the part of the orbit farther than earth from the sun. These atomic clocks show only the gravitational time dilation, due to the earth's gravitational (velocity) field.

In conclusion, Einstein's interpretation of the null results of the Michelson light anisotropy experiments is totally mistaken. But it was not simply a mistake. It has wasted a clear hint to unveil the nature and the origin of the gravitational physics. Michelson, Lorentz, Einstein and a whole generation of physicists have not realized that the null results of the light anisotropy experiments are exactly a clear and unmistakable signature of the real physical mechanism of gravity in action. Please see a description of this gravitational mechanism in Refs. [4]-[6]. However, besides this misstep, Einstein has left us the principle of the equivalence of gravitational and inertial effects, which is the key idea to understand the gravitational physics. Missing the clue of the null anisotropy of light, has at once dismissed a forthright implementation of this equivalence in the gravitational fields, forcing Einstein to invent the curved spacetime.

The Michelson experiments were performed within the non-inertial earth based laboratories. This non-inertial character entails implicit kinematical circumstances, related with the (spacedynamic) gravitational mechanism that Einstein should have taken into account in the interpretation of the Michelson experiments. It is obvious that, in order to get a reliable interpretation of the null results of these anisotropy experiments, it is absolutely essential to know first the true kinematical circumstances of the earth based laboratories.

The wrong interpretation of the Michelson experiments has lead to a wrong concept of space and thereby has closed the way to conceive the gravitational pull as a usual and genuine inertial effect. Because of this misstep, Einstein in his theory of gravity (GR) was forced to mock-up the gravitational dynamics in terms of the arcane and nonfigurative four-dimensional spacetime geometry, in which the paths of force-free mater bodies are modeled as geodesics into the geometry of spacetime. According to GR, the inertial references within gravitational fields are intrinsically free-falling as a consequence of the curved spacetime geometry. This however is not a physical explanation and actually is facing severe difficulties.

The spacetime geometry of GR imitates the gravitational dynamics and can explain many observed effects of the gravitational fields. Nevertheless, actually it cannot account for an increasing number of clear-cut experimental facts (please see Section 3). This shows that Einstein's TR does not disclose the true nature of space and of the gravitational physics. The TR symmetrizes space too much and the geometrical model of GR symmetrizes the gravitational physics too much, thereby eliminating small anisotropies that really exist and now, with the extremely precise atomic clocks in orbit, are being discovered. This however is not the most serious problem with GR. There is a much more serious shortcoming. GR, like the other current gravitational theories, cannot account neither for the observed gravitational dynamics within galaxies nor can it identify the physical cause of the accelerated expansion of the universe.

On the other hand, according to the spacedynamic gravitational mechanism [5] [6], the local inertial references are not free-falling, but are rotating round an overhead axis, due to the Keplerian velocity field of the QS ruling the inertial motion of matter and the propagation of light. This velocity field of the QS is clearly entailed by the recent experimental observations, achieved with the help of the tightly synchronized clocks in orbit. In the theory of spacedynamics, Einstein's equivalence of gravitational and inertial effects is genuinely implemented. The gravitational pull is conceived as an authentic centrifugal (fictitious) effect. The free-fall of bodies and the orbital motions of the planets and astronomical bodies in general are ruled by the principle of inertia. In addition, the circular orbital motions of the planets need not to be explained anymore, because it is space itself that so moves. Spacedynamics also predicts the observed non-Keplerian gravitational dynamics of the galaxies without the need of dark matter and appoints the physical cause, responsible for the accelerated expansion of the universe.

All the conventional experimental tests of the TR were made for very high velocities of atoms or elementary particles with respect to the earth based laboratories, in most cases these velocities are comparable with the velocity of light. According to recent experimental observations, described here in Section 3, the earth globe moves with respect to the QS only about a hundred of meters per second. This shows that earth is commoving with the QS in the velocity fields of the QS, generating the solar and the galactic gravitational fields. Therefore 
these velocity fields cannot produce observable effects within the earth based laboratories. Only the Keplerian velocity field of the QS round earth itself, creating the earth's gravitational field, is effective within the earth based laboratories. Moreover, the resolution of these experimental tests was much too low to evidence effects of the low velocity field of earth. Hence, these experimental tests are ambiguous because they can equally well be seen as tests of the effects, predicted by spacedynamics, due to motions with respect to the QS (Higgs condensate). The velocity field of the QS round earth circulates in the sense of the Moon's orbital motion and reaches $7.91 \mathrm{~km} / \mathrm{sec}$ on surface. Due to the slow rotation of Earth $(460 \mathrm{~m} / \mathrm{sec}$ at the equator), the velocity of the earth based laboratories with respect to the QS, at the sites of the experiments, is about $7.5 \mathrm{~km} / \mathrm{sec}$ due West (opposite to the circulation of the QS). The effect of this implicit velocity on light phenomena is in the order $10^{-10}$. Only very few light anisotropy experiments, using highly sensitive Michelson interferometers rotating in the earth based laboratory [12] were sensitive enough to barely detect effects of such low velocity of the earth based laboratories with respect to the QS (see Figure 1). Some Mössbauer experiments, testing the gravitational time dilation, the spectral red-shift [15] [16] too have clearly evidenced effects of the earth's gravitational field.

Note that the Lorentz contraction, due to relative velocity, cannot cancel the light anisotropy in the Michelson light anisotropy experiments, because the experiments have been made within earth based laboratories, in which the interferometer rests with respect to the observer. Moreover, the fact that the light anisotropy experiments, performed with highly sensitive Michelson interferometers, rotating within earth based laboratories, have detected the West-East anisotropy of nearly $8 \mathrm{~km} / \mathrm{sec}$, predicted by spacedynamics, proves that the Fitzgerald-Lorentz shortening of the interferometer arms, due to velocity with respect to the QS, does not occur. Add to this that to present date no direct experimental evidence is known concerning the Fitzgerald-Lorentz contraction. Visibly, the fact that these light anisotropy effects were much smaller than expected by the ether theories, due to the orbital and cosmic velocity of earth, has lead to the mistaken interpretation.

\subsection{The Phenomenon of Time Dilation Is Real}

The very notion of time arises from the evolution of the physical systems around us, which was already clear to the ancient Greek philosophers. Time has no meaning neither in the life of a stable atom in its ground state nor in the life of a charged isolated superconducting magnet in its persistent mode at very low temperature, because there is no evolution. In quantum mechanics the variables of time $t$ and of the total energy $E$ are conjugated together by the principle of uncertainty, which means that it is impossible to change one variable without affecting the other. Evolution of physical systems basically takes place by transformations of energy. The evolution of physical processes involves microscopic quantum transitions that are associated with characteristic electromagnetic oscillations. According to experiments and quantum mechanics, the period $T$ of atomic oscillations is inversely proportional to the involved variation of the total energy $\Delta E$ :

$$
T=h / \Delta E
$$

From the comparison between the periods of different oscillations arises the quantitative notion of time, the time rate.

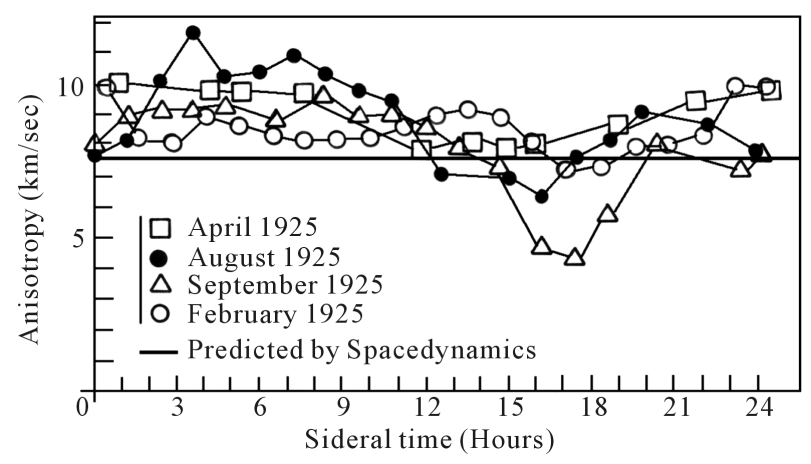

Figure 1. Ultimate and most complete light anisotropy data obtained by D. Miller. The small but systematic sinusoidal variation of the anisotropy along the 24 hours visibly is due to a cause not rotating with earth. 
Clocks count time in terms of the period $T$ of a classical or quantum oscillator. Actually the most precise clocks are the atomic clocks. They count time using as time standard the oscillation period of an electromagnetic (EM) cavity tuned to the very precise frequency of the hyperfine transition of gaseous alkali metal $\mathrm{Cs}$ or $\mathrm{Rb}$ atoms. The period $T$ of the EM oscillations of these atoms are go-return roundtrips of the EM field in the atomic structure, which involves mass and depends on the electromagnetic forces. Such oscillations are entirely analogous to the light (free field) go-return roundtrips and each specific oscillation is affected by the velocity of the laboratory with respect to the QS in the same proportion as light go-return roundtrips [2]. For roundtrips transverse to the motion of the laboratory, the relation between the period $T$ of the time standard (atoms or light), moving at velocity $v_{\mathrm{QS}}$ with respect to the QS is:

$$
T=\frac{T_{0}}{\left(1-v_{\mathrm{QS}}^{2} / c_{\mathrm{QS}}^{2}\right)^{1 / 2}}
$$

where $T_{0}$ is the characteristic period of the atom at rest with respect to the QS. Note that in this equation the velocity $v_{\mathrm{QS}}$ is not the relative velocity, but the velocity with respect to the QS and $c_{\mathrm{QS}}$ is the constant one-way velocity of light with respect to the QS. Due to the fact that the light go-return roundtrip and the go-return roundtrip of the time standard are affected in the same proportion by the velocity of the laboratory with respect to the QS, measuring the velocity of light by the light go-return roundtrip and clock method, necessarily gives always the same result, regardless the velocity of the laboratory with respect to the QS. Hence, the velocity of light, measured in this way, is an artifact of the improper measuring method. This method is improper because it measures the velocity of light by comparing the light go-return roundtrip time along a known distance with the roundtrip time of the time standard (atom) along a certain distance, by which the clock counts time and which are both affected in the same proportion by motion of the laboratory with respect to the QS. Due to the invariance of the velocity of light, measured in this way, such measurements can not reveal the state of motion of the laboratory with respect to the QS, which apparently only apparently corroborates Einstein's postulate of the intrinsic constancy of the velocity of light.

The crucial question now is: Is the light go-return roundtrip and clock method the only feasible way of measuring the velocity of light? In the age of the extremely precise and stable atomic clocks in orbit, synchronized all together to within $0.1 \mathrm{~ns}$ (time for light to travel $3 \mathrm{~cm}$ ), cannot the one-way velocity of light be measured precisely enough to falsify or validate the postulate of the constancy of light of the TR? Cannot the one-way anisotropy of light be measured precisely enough to falsify or confirm the small anisotropy of light with respect to the earth based laboratories, predicted by the present theory of spacedynamics?

\subsection{Anisotropy of the One-Way Velocity of Light}

In order to measure the velocity of light along one-way travels, there is need of having closely synchronized clocks, one at each end of the traveling distance so that the light signal along the one-way travel can be timekeeped. Synchronization of clocks located at different points in space certainly is a challenging issue. In Einstein's view [1] two clocks, resting with respect to each other at points $A$ and $B$ in space, can be synchronized by sending a light signal at time $t_{0}$ from $\mathrm{A}$ to $\mathrm{B}$, reflected back from B to A at time $t_{1}$ and arriving $\mathrm{A}$ at time $t_{2}$. If $t_{1}-t_{0}=t_{2}-t_{1}$, then clock $\mathrm{B}$ is synchronized with clock A. This equality is exactly the condition for the perfect isotropy of light, showing that Einstein effectively believed in the intrinsic isotropy of light within any possible inertial reference. Note that, despite this equality is established between one-way travels of light, the synchronization procedure is completed only after a tow-way go-return roundtrip. Einstein's clock synchronization method is conceived under the assumption that the velocity of light is intrinsically constant and isotropic. The only restriction is that the clocks must be resting with respect to each other in the observer's inertial reference. Hence, from this viewpoint, this synchronization procedure can work in all possible inertial references.

However, in the view of the present work, the velocity of light is constant and isotropic not with respect to all possible inertial references but only with respect to the QS that is circulating round each astronomical body according to a Keplerian velocity field, consistent with the local main astronomical motions. From this viewpoint, Einstein's synchronization method can succeed only if the system of the two clocks has no motion with respect to the QS along the line connecting A and B, because otherwise the velocity of light will be anisotropic along the path of the light signal and synchronization will be impossible. If the clocks move with respect to the QS transversely to line A-B so that this line remains parallel to itself, clock synchronization by Einstein's method 
can be achieved. In practice, if synchronization of the GPS clocks in orbit with the clock on ground is made when the satellites are nearly vertically above the ground station, so that the velocity of the EM signal between the station and the satellites is closely perpendicular to the velocity field of the QS creating the earth gravitational field, synchronization by Einstein's method may give enough precision.

In recent years experiments with the help of the very stable atomic clocks in orbit and synchronized collectively by Einstein's synchronization method to within $0.1 \mathrm{~ns}$ (time for light to travel $3 \mathrm{~cm}$ ), the one-way velocity of EM signals (light) between satellites has been measured very precisely. Especially clear-cut measurements of the one-way velocity of EM signals have been achieved in both senses with the help of the twin satellites of the Gravity Recovery and Climate Experiment (GRACE), both moving in a same low altitude polar orbit, in this way constituting an inertial reference very nearly common to both satellites. In order to measure microgravity effects, these twin satellites need be equipped with very stable atomic clocks, synchronized to better than $0.16 \mathrm{~ns}$. The position of these satellites was monitored by the GPS within $3 \mathrm{~cm}$. Observations have shown a clear one-way anisotropy of the EM signals (light) of nearly $8 \mathrm{~km} / \mathrm{sec}$ backward to the motion of the satellites [11]. This one-way anisotropy is exactly equal to the velocity of the satellites with respect to the earth non-rotating coordinate system (QS) and definitively breaks the believe on the intrinsic constancy and isotropy of light with respect to all possible inertial references. Please see Section 3.1 for details.

\subsection{Einstein's Postulate of the Intrinsic Constancy and Isotropy If Light}

In Einstein's view, the most precise method for measuring the velocity of light is timekeeping the go-return roundtrip of a light pulse along a known distance, using a precise clock. However, as pointed out in the above Section 4.2, the velocity of light, measured in this way, is an experimental artifact. Moreover, Einstein, encouraged by the nominally null results of the Michelson light anisotropy experiments, has postulated the intrinsic isotropic of light in all of the inertial references. However, recent experimental observations give evidence that the Michelson experiments gave nominally null results, not because of the intrinsic isotropy of light, however because earth very nearly rests with respect to the QS in the Keplerian velocity field generating the gravitational field of the sun, of the galaxy etc. Einstein's postulation of the intrinsic isotropy of light was the decisive misstep that has lead toward the actual impasse about the nature of space and the gravitational physics. It is the exact step that has excessively symmetrized space and the gravitational physics, eliminating small anisotropies that now are being discovered.

The experimental artifact of the velocity of light, measured by the light go-return roundtrip and clock method, plays a central role in all of Einstein's thought experiments. In physics, a constant parameter is very important to establish exact relations and to set equations. In his thought experiments, Einstein has cleverly explored the constancy and the isotropy of the velocity of light, that in his view is confirmed by the null results of the Michelson light experiments, to obtain corrections for time, length, mass, energy etc. as a function of the relative velocity between inertial references. Obviously Einstein could consider only relative velocities because, in his principle of relativity, absolute motions can not be defined. In all of these thought experiments, there is a hypothetically resting reference and a relatively moving reference. The observer in the resting reference is aware of the constancy and isotropy of light in his reference and makes conclusions from his point of view about the velocity of light, the rate of clocks, the change of mass, the lengths, the energy of radiation etc. in the relatively moving reference. However, strangely Einstein never has addressed him to the point of view of the observer in the moving reference.

Obviously, if it is the relative velocity that counts and if the velocity of light is intrinsically constant and isotropic in all references, there is reciprocal symmetry between the two observers and the moving observer can of course assume that his reference is the resting one and that the reference of the hypothetically resting observer is moving and then make conclusions about the happenings in this reference. If the assumptions of the TR are right, the formerly moving observer will of course come to conclusions symmetrically opposite to those of Einstein's hypothetically resting observer. This is the problem of the reciprocal symmetry between observers in the TR that leads to contradictions and many paradoxes that remain unsolved or badly resolved.

In the view of the present work, a resting reference must effectively be stationary with respect to the QS and a moving reference is really moving with respect to the QS. Therefore in the present theory of spacedynamics, there is no reciprocal symmetry between observers. Note however that, within gravitational fields, the QS is moving according to a Keplerian velocity field (Equation (1)) round each body, consistently with the local main 
astronomical motions. Therefore, within gravitational fields, in order to a reference to be resting with respect to the QS, it must be commoving with the QS in the Keplerian velocity field creating the gravitational field (circular equatorial orbit). Earth is commoving with the QS in the solar Keplerian velocity field, however the earth based laboratories are not commoving with the QS in the earth's Keplerian velocity field because earth rotates much slower than the QS. The velocity of the earth based laboratories with respect to the QS is somewhat lower than $8 \mathrm{~km} / \mathrm{sec}$. All the experiments effectively performed to test the predictions of the TR, were made within earth based laboratories, however for particles having velocities many orders of magnitude larger than $8 \mathrm{~km} / \mathrm{sec}$. For such high velocities, the velocity of the testing bodies with respect to the laboratory is closely equal to the corresponding velocity with respect to the local QS. Although these experimental tests are frequently claimed to confirm the predictions of the TR, within their experimental precision, these experiments cannot distinguish between the effects, due to the relative velocity (with respect to the earth based laboratory) from the effects, due to the velocity with respect to the local QS. Hence, these experiments can equally well be claimed to corroborate the predictions of the theory of spacedynamics. In order to really test the predictions of the TR, it would be necessary to perform tests between inertial references both moving with respect to earth at high and opposite velocities.

\subsection{Einstein's Justification of Time Dilation}

In order to demonstrate that relative velocity causes time dilation, Einstein imagined an observer moving with his clock at a relative velocity $v_{r}$ with respect to the reference of a hypothetically resting observer. In Einstein's view, if the velocity of light is isotropic in the reference of the resting observer, then, in the view of this resting observer, light can no longer be isotropic with respect to the moving observer, because otherwise the postulate of the constancy of the velocity of light in the reference of the resting observer would be broken. Note that, in the present theory of spacedynamics, it is exactly this judgment of the resting observer that is refuted. From the viewpoint of spacedynamics, despite Earth and Mars are relatively moving with respect to each other, both planets are locally resting with respect to the QS in the solar velocity field and hence the (one-way) velocity of light is really isotropic with respect to both planets. Obviously, in order to this be possible, the velocity of light must inexorably change as a function of position. This variation of the velocity of light follows exactly the variation of the velocity of the QS in the Keplerian velocity field creating the gravitational field of the sun.

In his paper on Electrodynamics of Moving Bodies [1] Einstein textually writes that, in the opinion of the resting observer, the velocity of light with respect to the moving observer from the back side is $c-v_{r}$, while from the front side it is $c+v_{r}$. Hence, in the view of this resting observer, the velocity of light in the moving reference is anisotropic and light go-return roundtrips within the moving reference, transversely to the motion must take a longer time $t=t_{0}\left(1-v_{r}^{2} / c^{2}\right)^{-1 / 2}$, where $t_{0}$ is the roundtrip time in the resting reference. Therefore, in the view of this resting observer, the velocity of light, measured by the moving observer, using the method of light go-return roundtrips and clock, can be equal to $c$ only if the rate of the clock in the moving reference runs slower by exactly this same amount for any value of the relative velocity. This conclusion is a perfect practical realization of the assertion in Section 4.2 that the velocity of light, measured by the light go-return roundtrip and clock method, is an artifact of the measuring method and not a principle of nature. In fact Einstein introduces additionally that the velocity of light is perfectly isotropic with base in the null results of the Michelson light anisotropy experiments so that transverse and longitudinal roundtrips in the moving reference become identical, which has as consequence the shortening of the distances along the motion.

According to the present work, the one-way velocity of light can be isotropic in the reference of the hypothetically resting observer, only if this reference really is stationary with respect to the QS. In this case, the observer relatively moving at the same place necessarily is really moving with respect to the QS and the (one-way) velocity of light effectively is anisotropic with respect to him. Therefore the light go-return roundtrips as well as the roundtrips of the time standard, by which the clock counts time, take both a proportionally longer time. Curiously, Einstein assumes in all of his thought experiments that one observer is hypothetically resting and makes conclusions about the happenings in the moving reference from his point of view. However, in the view of the present work the earth based laboratories, in which the experimental tests of the TR have been made, really are very nearly stationary with respect to the QS and so closely accomplish the kinematical circumstances of Einstein's hypothetical resting observer. Hence, within these circumstances, high velocity with respect to the laboratory has practically the same value as the velocity with respect to the QS and both theories predict effects that, 
within the experimental precision, are of the same magnitude. This shows that, despite the radically opposite assumptions in the present work (real QS ruling the inertial motion of matter and the propagation of light), in the effectively tested practical situations (within earth based laboratories) the conclusion about time dilation and clock synchronization, within the experimental precision, are the same. Clearly the wrong interpretation of the null results of the Michelson light anisotropy experiments is responsible for Einstein's peculiar view about these physical effects.

\subsection{The Reciprocal Symmetry, an Open Problem in the TR}

In Einstein's thought experiment [1] about time dilation, due to relative velocity, the reciprocal symmetry between relatively moving observers leads to conflicting conclusions between observers and many paradoxes. The observer in the moving reference can of course measure the velocity of light in his reference by the method of two-way light go-return roundtrips and his clock and find that, contrarily to the opinion of the resting observer, the velocity of light coming from his back or from his front sides have exactly the same value and hence that in his reference light is isotropic along the motion. What in fact the moving observer is doing by using this method is comparing the average velocity of light in roundtrips along his apparatus, computing it first for a forward followed by a backward light travel along the same distance and then inverting the sequence and calculating the average for first a backward and then a forward travel. Obviously the averages must give the same value because they are made for exactly the same two light transit times by only interchanging their time sequence. The origin of the discrepancy between the opinions of the resting and the moving observer arises from the fact that the resting observer comes to his conclusion by a simple logic in terms of the constancy of light in his reference, while that of the moving observer is the result of a misleading measuring method, based in the average velocity of light for two-way light roundtrips. These conflicting conclusions expose the ambiguity and the fallacy of the postulate of the intrinsic isotropy and constancy of light. In the view of the present work, Einstein's conclusion about the slowness of the moving observer's clock is correct if the resting observer is effectively resting and the moving observer is effectively moving with respect to the QS. In case the hypothetically resting observer is moving with respect to the QS, his conclusions about the happenings in the relatively moving reference almost certainly are wrong.

Einstein has related time dilation to relative velocity. If time dilation were effectively due to relative velocity, a moving observer obviously could assume that his own reference is stationary and that the hypothetically resting observer is moving. Then by a similar reasoning, as that of Einstein's hypothetical resting observer could reach to the conclusion that the clock of the hypothetically resting observer is running slow. This is another ambiguity of the TR, now about time dilation due to the reciprocal symmetry between observers. This ambiguity has lead to the famous twin paradox. The twin paradox never has been adequately resolved. Einstein and other people have claimed that in the case of the twin paradox, the traveling twin necessarily must change inertial reference in various stages of his roundtrip travel, which involves accelerations and that these accelerations suppress the reciprocal symmetry between the twins and thereby are ultimately responsible for slowing down the aging of the traveling twin. More recently some authors [17] allege that clocks resting in gravitational fields too run slow because of their (implicit) upward acceleration and for this reason the GPS clocks that together with earth are free falling in the solar gravitational field, should not show the gravitational time dilation due to the solar gravitational potential. Nevertheless, from this viewpoint, the GPS clocks are also free falling in the earth's gravitational field and notwithstanding clearly show the gravitational time dilation due to the earth's gravitational field (please see Section 2.2 in Ref. [5] [6] for details). To this should be added that acceleration has been tested by Muon decay within cyclotrons up to $10^{19} \mathrm{~m} / \mathrm{sec}^{2}$ with the clear-cut conclusion that accelerations do not cause time dilation [18]. The experimental observations, described here in Section 3 prove that time dilation is not caused by relative velocity, however by velocity with respect to the QS in free space and by implicit velocity with respect to the QS within gravitational fields. This puts an end to the reciprocal symmetry and hence to the impasses created by this ambiguity.

\subsection{The Gravitational Time Dilation}

In GR (pgs. 101-105 of Ref. [1]) Einstein devised a thought experiment in which an atom in the gravitational field absorbs a photon of a well defined energy making a transition to a definite excited state. Due to the mass associated with energy in this higher energy state its mass is a little bit larger than in the ground state and therefore, 
if elevated in the gravitational field the excited atom will correspondingly store a larger potential energy. At the higher altitude, the atom may relax emitting a photon with a correspondingly larger energy, which is the excitation energy of the atom at the higher altitude. Hence, the excitation energy (frequency) of an atom at higher altitude is larger than of the same atom at lower altitude. Einstein rightly concluded from this simple thought experiment that the (negative) gravitational potential lowers the characteristic frequencies of atoms. This corresponds to saying that the energy spacing between the quantum energy levels of the atomic structure decreases or, what is the same thing, the mass involved in the atomic oscillations increases on going to lower altitudes in the gravitational field. This is the gravitational time dilation, the gravitational slowing of clocks and the gravitational spectral red-shifts. Actually Einstein's gravitational time dilation and the gravitational spectral red-shifts are well confirmed experimental facts. However, although the conclusion is correct, the explanation in terms of the gravitational potential is wrong. According to the recent experimental observations, described in Section 3.2, it is not the gravitational potential that causes the gravitational time dilation of clocks, resting with respect to ordinary space coordinates within a gravitational field, but the implicit velocity of these clocks with respect to the QS, due to the Keplerian velocity field creating the gravitational field. It is strange that the TR needs to involve two completely different causes, the relative velocity and the gravitational potential, producing the same effect of time dilation.

In the present theory of spacedynamics, the cause of time dilation is unified. Clocks run slow neither because of the relative velocity nor because of the gravitational potential. They run slow because of their velocity with respect to the QS. A clock fixed with respect to the ordinary space coordinates within a gravitational field, is implicitly moving with respect to the QS at a velocity $-V_{\mathrm{QS}}$ that is the negative of Equation (1). This velocity is implicit because it cannot be specified with respect to the ordinary space coordinates. Within gravitational fields, it is the QS, the ultimate reference for rest and for motions, that is moving through the fixed clocks. In this condition, the roundtrip time period $T$ of the time standard (oscillator), by which the clock counts time, is longer than $T_{0}$ of an oscillator resting with respect to the QS, according to the well known formula:

$$
T=T_{0}\left[\frac{1}{1-V_{\mathrm{QS}}^{2} / c_{\mathrm{QS}}^{2}}\right]^{x}=T_{0}\left[\frac{1}{1-(\gamma M / r) / c_{\mathrm{QS}}^{2}}\right]^{x}
$$

where $x=1$ for longitudinal roundtrips and $x=0.5$ for transverse roundtrips.

Experiments, described in Subsection 3.1, show that light go-return roundtrips within earth based laboratories are a little bit anisotropic $(8 \mathrm{~km} / \mathrm{sec})$. The effect of this anisotropy on the EM phenomena is in the order of (about $10^{-10} \mathrm{sec} / \mathrm{sec}$ ) along the East-West direction. This means that East-West roundtrips of EM signals on earth take a little bit more time than North-South roundtrips. Actual atomic clocks are precise enough to test this prediction. However, almost certainly atomic clocks will not show such anisotropy because the energy levels of atoms should not be anisotropic, so that the exponent $x=1$, corresponding to the lower energy, may prevail. However, such anisotropy could well be observable by Light go-return clocks or highly sensitive Michelson light anisotropy experiments.

\subsection{The Relativity of Simultaneity}

According to Einstein's criterion, two events taking place at different points in homogeneous space are simultaneous if light signals, emitted by them, reach an observer at the midpoint between them at the same time. However, in the opinion of another observer, moving along the right line connecting the two events and passing by the midpoint exactly at the instant the events occur, these same events are not simultaneous [1]. The reason is that, from the viewpoint of the resting observer, during the time the signals are traveling from both ends toward the midpoint, the moving observer will have moved away from this midpoint and so will see the light signal from his front event before the one from the rear event. Note that in this thought experiment Einstein makes use of the one-way velocity of light. He assumes that the velocity of light, that is isotropic by definition with respect to the hypothetically resting observer at the midpoint, is no longer isotropic with respect to the moving observer. Otherwise, the moving observer too would see the events simultaneously. However, the moving observer can of course measure the velocity of light by the two-way light go-return roundtrip and clock method and find that the velocity of light coming from his back or from his front side have exactly the same value $c$ and that consequently the velocity of light, along the motion is isotropic with respect to him and hence that it can no longer be iso- 
tropic in the reference of the hypothetical observer at the midpoint who passed the midpoint in his reference exactly when the events occurred. Therefore, in the opinion of the moving observer the hypothetically resting observer must see first the light from the back event.

From the present spacedynamics viewpoint, light propagates at a well defined one-way velocity $c_{\mathrm{QS}}$ not with respect to all possible inertial references, however only with respect to the QS that rules its propagation. From this viewpoint, Einstein's criterion for simultaneity is correct only if the laboratory, in which the experiment is being made, has no velocity with respect to the QS along the line connecting the two events. If this is true and the two events are simultaneous in the opinion of an observer, resting with respect to the QS at the midpoint between them, then in the opinion of an observer, moving along the line connecting the events and passing by the midpoint at the instant the events occur, the events will not appear to be simultaneous and moreover the one-way velocity of light is not isotropic along his motion (no reciprocal symmetry). However, this moving observer can measure the anisotropy of light in his reference by a highly sensitive Michelson interferometer or can use a one-way technique and thereby find the velocity $v_{\mathrm{QS}}$ of his reference with respect to the QS and then, by a little calculation in terms of the effective velocity of light $\boldsymbol{c}_{\text {eff }}=\boldsymbol{c}_{\mathrm{QS}}+\boldsymbol{v}_{\mathrm{QS}}$ with respect to his reference, find the true temporal separation of the events.

\subsection{The Relativity of Mass}

From the viewpoint of the present work the change of the mass of matter bodies, due to motion with respect to the QS can be obtained by physical arguments directly from the corresponding change of the period of an oscillator. In linear harmonic oscillations the oscillation period $T$ is directly proportional to the square root of the mass involved in the oscillation $T=2 \pi(\mathrm{m} / \mathrm{k})^{1 / 2}$, where $k$ is a characteristic constant for each specific oscillator. Oscillations of atoms are lumped EM oscillations that involve collectively the electrons that have mass. Due to this involved mass, atoms can emit radiation with wavelength much longer than the size of the atom. Oscillations in fact constitute a very precise method for measuring changes of mass. The oscillation period $T$ of time standards (atoms) has been experimentally verified to increase when atoms move with respect to the laboratory at high velocity, which in fact is velocity with respect to the QS (please see Ref. [2] and also Equations (3) and (4) above). However, if the oscillation period increases, the mass involved in the oscillation, must increase proportionally according to the equation:

$$
T=\frac{T_{0}}{\left(1-v_{\mathrm{QS}}^{2} / c_{\mathrm{QS}}^{2}\right)^{1 / 2}}=2 \pi\left(\frac{m / k}{1-v_{\mathrm{QS}}^{2} / c_{\mathrm{QS}}^{2}}\right)^{1 / 2}
$$

Note that in this equation $v_{\mathrm{QS}}$ is the velocity with respect to the QS. This equation is identical to the one obtained by Einstein for the mass of a slowly accelerated electron in the inertial reference of the observer.

\subsection{Addition of Velocities}

Velocity, by definition, is the ratio between the distance traveled by a body and the corresponding time interval of the travel in the limit of infinitesimal traveling distances. According to the TR, distances decrease and time intervals increase as a function of the relative velocity. Moreover, the velocity of light $c$ is a limiting velocity that nothing can exceed. In the Large Hadrons Collider (LHC) in Geneva, protons, speeding oppositely both at very nearly the velocity of light, are frontally smashed. According to the TR the relative velocity between these protons is lower than the velocity of light. Although this seems illogic, it must be kept in mind that, in the TR, the addition of velocity cannot be made by vector addition, but must be seen from the viewpoint of measurements.

According to the present work, the one-way velocity of light has a well defined value $c_{\mathrm{QS}}$ with respect to the QS and this too is a limiting velocity that nothing can exceed, because this velocity is the maximum velocity with which the QS can propagate perturbations. This is analogous to the propagation of perturbations in the atmosphere. The atmosphere has a limiting velocity for propagating perturbations of the atmospheric pressure. Measurement of the one-way velocity of particles may be made by timekeeping the one-way travel, using two clocks separated a distance $L_{0}$ and synchronized in common view with a standard clock that rests with respect to the QS and displays proper time. If the inertial reference of the two synchronized clocks moves with respect to the QS at velocity $v$ along the longitudinal direction the rate of the two clocks decreases equally according to 
Equation (3). If then the observer, moving with the clocks, measures the one way velocity of light parallel to the motion, he will find $c^{\prime}=\left(c_{\mathrm{QS}}-v_{\mathrm{QS}}\right) /\left(1-v_{\mathrm{QS}}^{2} / c_{\mathrm{QS}}^{2}\right)^{1 / 2}$ for parallel light pulses and $c^{\prime}=\left(c_{\mathrm{QS}}+v_{\mathrm{QS}}\right) /\left(1-v_{\mathrm{QS}}^{2} / c_{\mathrm{QS}}^{2}\right)^{1 / 2}$ for anti-parallel light pulses. Analogously, for a particle moving along the parallel longitudinal direction at velocity $V$ with respect to the QS, he will find $v^{\prime}=\left(V_{\mathrm{QS}}-v_{\mathrm{QS}}\right) /\left(1-v_{\mathrm{QS}}^{2} / c_{\mathrm{QS}}^{2}\right)^{1 / 2}$ and $v^{\prime}=\left(V_{\mathrm{QS}}+v_{\mathrm{QS}}\right) /\left(1-v_{\mathrm{QS}}^{2} / c_{\mathrm{QS}}^{2}\right)^{1 / 2}$ for anti-parallel motion of the particle. The fact that the measured velocity can be larger than $c_{\mathrm{QS}}$ is due to the fact that these moving clocks do not measure proper time.

\subsection{The Problem with the Lorentz Transformations}

In order to find general rules for transforming results of measurements between relatively moving observers, Einstein made use of another clever thought experiment [1]. He imagined a resting coordinate system $(x, y, z)$ in which the velocity of light is isotropic and a reference $(\chi, \eta, \zeta)$ moving with respect to the resting reference at a velocity $v$. Then from the origin of the moving reference at time $\tau_{0}$ of the clock in the moving coordinate system a light pulse is emitted along the common axis of $\mathrm{X}$ and at a time $\tau_{1}$ is reflected back at a point fixed in the moving reference, that corresponds to $x^{\prime}=x-v t$ with respect to the resting reference and arriving at the origin of the moving reference at time $\tau_{2}$. Applying the postulate of the intrinsic isotropy of light in the moving reference, he asserts that the instant at which light is reflected at $x^{\prime}$ is given by $\tau_{1}=\left(\tau_{0}+\tau_{2}\right) / 2$. He then calculates $\tau, \chi, \eta$ and $\zeta$ in terms of $t, x, y$ and $z$ of the resting reference, using the argument that, if in the opinion of the observer in the resting reference light is isotropic, then, in the viewpoint of the resting observer, it can no longer be isotropic in the moving reference.

Note that first Einstein finds the equation $\tau_{1}=\left(\tau_{0}+\tau_{2}\right) / 2$ with base in the intrinsic isotropy of light in the moving reference and then asserts with base in the isotropy of light in the resting reference, that, in the opinion of the resting observer, light can no longer be isotropic in the moving reference. The conflicting opinions between the moving and the resting observers clearly arise because of the postulate of the intrinsic isotropy of light with respect to all possible inertial references, which arises from the mistaken interpretation of the null results of the Michelson light anisotropy experiments and leads to the reciprocal symmetry between the observers. In order to find explicit general rules for transforming results of measurements between inertial references (Lorentz transformations), Einstein also imagined another thought experiment. In this imaginary experiment, two observers, one in a hypothetically resting coordinate system and another one in a coordinate system moving with respect to the first along the common axes of $\mathrm{X}$ with relative velocity $v$. Then at the exact instant the origin of the moving observer's coordinate system passes the origin of the resting system a light pulse is emitted in all directions from the coincident origins. According to the postulate of the intrinsic constancy and isotropy of light with respect to all inertial references, the light pulse must expand in both coordinate systems according to spherical fronts about the origin of each coordinate system. Within the hypothetically resting reference the velocity of light has the value $c$ and is isotropic by definition. It expands according to a spherically symmetric wave front:

$$
x^{2}+y^{2}+z^{2}=c^{2} t^{2}
$$

According to Einstein, in the moving coordinate system light may expand according to a spherically symmetric wave front too:

$$
x^{\prime 2}+y^{\prime 2}+z^{\prime 2}=c^{2} t^{\prime 2}
$$

However, in the view of the resting observer, if light is isotropic in his coordinate system, then it can no longer be isotropic within the moving coordinate system. Hence, light roundtrips along the longitudinal direction may take a little bit more time than along the transverse directions. If true, an observer in the moving coordinate system may detect this anisotropy. With base in the null results of the Michelson light anisotropy experiments and the postulate of the intrinsic isotropy of light, Einstein has enforced the perfect isotropy of light in the moving reference and hence perfect spherical symmetry of the outgoing light fronts in both the hypothetically resting and the moving coordinate systems. On finding the rules for transforming length and times from one coordinate system to the other, this cunning imposition has generated a contraction factor for the lengths along the longitudinal direction (axis of $\mathrm{X}$ ) as a function of the relative velocity that is present in the well known Lorentz transformations of coordinates. This length contraction along the relative velocity, is an artifact introduced by imposition of the perfect isotropy of light with respect to all possible inertial references.

In the view of the present work, light can be isotropic only within a reference, stationary with respect to the QS, ruling the inertial motion of matter and the propagation of light, that is, resting with respect to it. Within any 
reference moving with respect to the QS, light necessarily is anisotropic as clearly demonstrated by recent measurements of the one-way velocity of light (see Section 3.1). This one-way anisotropy of light frontally contradicts the postulate of the intrinsic isotropy of light. Moreover, anisotropy experiments with highly sensitive Michelson interferometers also have revealed the small East-West light anisotropy, predicted by the present theory of spacedynamics [5] [6]. Note that these anisotropies are very small because earth is commoving with the QS in the Keplerian velocity field generating the gravitational fields of the sun and of the galaxy. The experimental observation of these small anisotropies demonstrates that true FitzGerald-Lorentz contraction of the lengths of bodies, due to motion with respect to the QS, does not occur and hence that the Lorentz transformations, obtained by imposing the intrinsic isotropy of light, are contrived. Besides this, note that to present date no direct experimental evidence has been obtained for the FitzGerald-Lorentz contraction. As asserted previously, the postulate of the intrinsic isotropy of light was exactly the critical misstep that has lead to abandonment of the search for the very small anisotropies in the light phenomena within gravitational fields that soon or late would have lead to the discovery of the spacedynamic nature of the gravitational physics.

\subsection{The Equivalent of Mass and Energy}

By far the most interesting result of the TR is the mass-energy equivalent. In order to find this, Einstein used another thought experiment in which a radiation source, resting in the $(x, y, z)$ coordinate system of a resting observer emits two equal light (energy) pulses $\frac{1}{2} L$ exactly in opposite directions (along the axis of $\mathrm{X}$ ) so that the source remains resting in the resting inertial reference. However, to an observer in a coordinate system $\left(x^{\prime}, y^{\prime}, z^{\prime}\right)$ moving at velocity $-v$ along the common axis of $\mathrm{X}$, the source will be moving with velocity $+v$ along $+x^{\prime}$ porting a kinetic energy $K_{0}$. To the moving observer the energy $\frac{1}{2} L$ of the radiation pulses along $+x^{\prime}$ will be larger than that along $-x^{\prime}$, because of two effects: The Doppler shift $L(1 \mp v / c)$ and the time dilation of the source $L\left(1-v^{2} / c^{2}\right)^{-1 / 2}$, due to the relative velocity $v$.

$$
E=\frac{1}{2} L\left[\frac{1-v / c}{\left(1-v^{2} / c^{2}\right)^{1 / 2}}+\frac{1+v / c}{\left(1-v^{2} / c^{2}\right)^{1 / 2}}\right]=\frac{L}{\left(1-v^{2} / c^{2}\right)^{1 / 2}}
$$

where the last term is the energy taken up by the radiation from the body in the moving reference, that is larger than that in the resting reference. As in the moving reference this energy goes in the positive $x^{\prime}$ direction, the kinetic energy of the body in the moving reference decreases.

$$
K_{0}-K=L\left[\frac{1}{\left(1-v^{2} / c^{2}\right)^{1 / 2}}-1\right] \approx \frac{1}{2} \frac{L}{c^{2}} v^{2}
$$

where $K_{0}$ is the initial kinetic energy of the body in the opinion of the moving observer and $K$ is the kinetic energy after the emission. The last equality is an approximation for low velocity, where $L / c^{2}=m$ is the mass associated with the radiation that may be written:

$$
E=m c^{2}
$$

\subsection{The Total Energy of a Moving Mass}

The total energy of a mass can be found integrating the work $(W)$ in terms of the potential done by the force $F$ accelerating a mass $m$, correcting $m$ and the corresponding momentum $p$ as a function of the velocity $v_{\mathrm{QS}}$ with respect to the $\mathrm{QS}\left(m=m_{0} /\left(1-v_{\mathrm{QS}}^{2} / c_{\mathrm{QS}}^{2}\right)\right)$, as found for transverse oscillations in Section 4.9:

$$
W=\int_{0}^{t^{\prime}} F v_{\mathrm{QS}} \mathrm{d} t=\int_{0}^{t^{\prime}} v_{\mathrm{QS}} \frac{\mathrm{d} p}{\mathrm{~d} t} \mathrm{~d} t=\int_{0}^{p^{\prime}} v_{\mathrm{QS}} \mathrm{d} p=\left[v_{\mathrm{QS}} p\right]_{0}^{v_{\mathrm{QS}}^{\prime}}-\int_{0}^{v_{\mathrm{QS}}^{\prime}} p \mathrm{~d} v=m_{0} c_{\mathrm{QS}}^{2}\left[\frac{1}{1-v_{\mathrm{QS}}^{2} / c_{\mathrm{QS}}^{2}}\right]_{0}^{v_{\mathrm{QS}}^{\prime}}
$$


Inserting the limits, we get:

$$
W=\frac{m_{0} c_{\mathrm{QS}}^{2}}{1-v_{\mathrm{QS}}^{2} / c_{\mathrm{QS}}^{2}}-m_{0} c_{\mathrm{QS}}^{2}
$$

where the first term at the right hand side is the total effective energy $E_{\mathrm{QS}}=m c_{\mathrm{QS}}^{2}$ and $m=m_{0} /\left(1-v_{\mathrm{QS}}^{2} / c_{\mathrm{QS}}^{2}\right)$. The second term is the resting energy $E_{0}=m_{0} c_{\mathrm{QS}}^{2}$. Equating the work $W$ to the effective kinetic energy $K_{\mathrm{QS}}$ of the mass, this expression can be written in the form:

$$
m c_{\mathrm{QS}}^{2}=K_{\mathrm{QS}}+m_{0} c_{\mathrm{QS}}^{2}
$$

which has the same form as the well known expression for the total relativistic energy, except that it is not for relative velocity $v$ between inertial references but for the velocities with respect to the $\mathrm{QS}\left(v_{\mathrm{QS}}\right)$. In the view of the present work $m_{0} c_{\mathrm{QS}}^{2}$ is the energy necessary to excite the particle in the Higgs QS, and $K_{\mathrm{QS}}$ is the additional energy, related with the additional phase distortions in the $\mathrm{HC}$ to set the particle into motion (propagation). The excited particle as well as the additional phase distortions in the $\mathrm{HC}$ are persistent modes and therefore are perfectly conserved quantities. The $K_{\mathrm{QS}}$ and $m_{0} c_{\mathrm{QS}}^{2}$ energy terms are mutually independent and must be treated as orthogonal quantities (four-vectors in the language of the TR). Therefore $E_{\mathrm{QS}}^{2}=K_{\mathrm{QS}}^{2}+m_{0}^{2} c_{\mathrm{QS}}^{4}$ or in terms of the momentum $m^{2} c_{\mathrm{QS}}^{4}-m_{0}^{2} c_{\mathrm{QS}}^{4}=c_{\mathrm{QS}}^{2} p_{\mathrm{QS}}^{2}$ :

$$
E_{\mathrm{QS}}^{2}=c_{\mathrm{QS}}^{2} p_{\mathrm{QS}}^{2}+m_{0}^{2} c_{\mathrm{QS}}^{4}
$$

\subsection{Summary and Conclusions to Section 4}

The difficult aspects of the TR remember the difficulties of Ptolemy's geocentric model with the astronomical motions. Analogously as the heliocentric system allowed astronomers to understand the apparently irregular motions of the planets, in terms of the simple circular Keplerian orbital motions round the sun, so does now spacedynamics allow us to understand the apparently magic relativistic effects and the observed gravitational dynamics in terms of the circulation field of the QS, ruling the inertial motion of matter and the propagation of light, round each astronomical body, according to a Keplerian velocity field consistent with the local main astronomical motions.

Recent experimental observations, described in Section 3, demonstrate that Einstein's interpretation of the null results of the Michelson light anisotropy experiments is wrong and that the fundamental assumptions of the TR about the velocity of light are false. The wrong interpretation of the Michelson experiments has lead to a wrong conception about the nature of space and matter. In the view of the QFT, underlying the SEPM, space is filled up with the Higgs condensate, which is a very powerful quantum space (QS) ruling the inertial motion of matter and the propagation of light. Moreover, the recent experimental observations also demonstrate that this $\mathrm{HC}$ is moving round earth, round the sun and round each astronomical body throughout the universe according to Keplerian velocity field, consistent with the local main astronomical motions. Within this spacedynamics earth is very closely resting with respect to the QS. Moreover, recent experimental observations demonstrate that the velocity of light has a well defined value and is isotropic with respect to the local moving Higgs-QS and not with respect to all inertial references. This predicts the nominally null results of the Michelson light anisotropy experiments.

Amazingly, in spite of the mistaken assumptions about the nature of space and the velocity of light, Einstein has obtained several effects, due to relative velocity that seem to match well the experimental observations and usually are claimed to validate the TR. However, the experimental tests of the TR were all performed within earth based laboratories. In the view of spacedynamics, these earth based laboratories are very closely stationary with respect to the QS, ruling the inertial motion of matter and the propagation of light. Hence, these laboratories accomplish very closely the kinematical circumstances postulated by Einstein for the resting observer in all of his thought experiments. Therefore, these experiments do not really test the predictions of the TR, but simply show the effects due to motions of the earth based laboratories with respect to the local QS. To claim that these experiments confirm the predictions of the TR is dishonest. It is like postulating that the atmospheric pressure is a universal constant throughout the universe and intending confirmation by measurements round the world only at the ocean level. 


\section{Possible Unification of General Relativity and of the Quantum Theory}

The new conception of space, matter and gravitation of the present work, in which the Higgs condensate (HC) plays the role of the QS, associates together the central idea of Einstein's GR, according to which the gravitational pull on us is an inertial pull and the idea of QFT underlying the SEPM, according to which the HC is a QS that rules the inertial motion of matter and the propagation of light. In the new conception, gravitation is not due to geodesic (inertial) motion within Einstein's curved four-dimensional spacetime. It also is not the result of a central field of gravitational forces, created by the quantum exchange interaction mediated by gravitons, as proposed in quantum gravity. In the new conception Einstein's curved four-dimensional spacetime is replaced by a macroscopic velocity field of the real QS, ruling the inertial motion of matter and the propagation of light, round each astronomical body, consistently with the local main astronomical motions. The gravitational acceleration is the result of inertial motion of matter bodies and light within the moving and warping real QS in the ordinary three dimensions according to a Keplerian velocity field $\boldsymbol{V}(r)=(\gamma M / r)^{1 / 2} \boldsymbol{e}_{\phi}$. This Keplerian velocity field is the quintessence of the gravitational fields that is shown in Refs. [4]-[6] to appropriately create the gravitational dynamics on earth, in the solar system and also the non-Keplerian gravitational dynamics of the galaxies without the need of dark matter.

\section{Universality of the Laws of Physics throughout the Universe}

Many observations indicate that, excepting only very strong gravitational fields, the laws of physics are the same throughout the universe and also with time. According to the present view the motion of the real QS is closely consistent with the astronomical motions throughout the universe. Hence, this universality of the laws of physics arises straightforwardly because the astronomical bodies essentially trace out very closely the motion of the QS itself (Higgs condensate) creating the respective gravitational fields. Hence, each astronomical body is closely stationary with respect to the local moving QS that rules the propagation of light and the inertial motion of matter. Therefore the velocity of light is the same and is isotropic with respect to all these bodies, which explains the null results of the Michelson light anisotropy experiments and also implies that clocks commoving with these bodies run all closely synchronous and show all closely the universal proper time. This must have been so since the formation of the first stars and galaxies. The universality of the laws of physics thus is a straightforward consequence of the fact that the physical phenomena taking place on Earth and in all these worlds evolve locally under closely the same kinematical circumstances (rest) with respect to the local QS. This spatial invariance of the laws of physics is clearly not the same thing as Lorentz invariance. In order to unambiguously verify the Lorentz invariance, the laws of physics would have to be exactly the same in all the different inertial references at the same place.

The superconducting condensate is physically anchored to the superconducting material. In the view of the TR, it is not Lorentz invariant because it has a preferred reference that is the superconducting material. The Higgs condensate, the QS has no such preferred reference. Moreover, if all the elementary particles get their mechanical properties from the Higgs condensate (HC), they cannot represent a reference for the local motion of the HC. Contrarily, the moving HC, the Higgs-QS is the local ultimate reference for rest and for motion of matter and light. Along its motions this QS carries with it all the ultimate and locally absolute reference for rest and for motion of matter and for all the material phenomena. This will say that the locally moving HC, the QS is necessarily itself the local preferred reference for rest and or motion of matter. Hence, its motion is completely innocuous to the local physical phenomena within a laboratory commoving with the QS. The motion of the HC is a gauge invariant. This assures the close sameness of the laws of physics on all natural astronomical bodies throughout the universe because they all very nearly rest with respect to the QS. On the other hand, the expansion of the universe is expansion of the QS itself that however does not expand bound systems like galaxies. If the BE condensation of the Higgs bosons is actually going on, the rate of this condensation may be larger at the colder intergalactic spaces. Moreover, as the minimum potential energy, in the Mexican sombrero potential, corresponds to a limiting volumetric density of the Higgs condensate, minimization of the energy of the QS may lead to expansion of the QS in these colder intergalactic regions. The observed Hubble spectral redshifts of radiation from galaxies show that on the overall the galaxies are reseeding from each other. However, these redshifts are not usual Doppler shifts. They are due to stretching of the wavelengths of radiation as a function of time, due to expansion of the QS (their medium of propagation) along their path.

Now several experimental observations (see Section 3) prove that within gravitational fields the QS moves 
round the astronomical bodies according to a Keplerian velocity field, consistent with the local main astronomical motions, thereby creating the gravitational fields. Hence, within gravitational fields, the preferred reference is a different one at each point of space. Only with few exceptions, each natural satellite, each planet of the solar system, each star in the galactic disk and each galaxy represent very closely the local preferred reference. All these bodies very closely rest with respect to the local moving QS. The observation that physical events on Mars or on very distant galaxies evolve the same rate as on Earth, has often been claimed to prove that the laws of physics are Lorentz invariant, that is, are not affected by the visible relative velocity of all these bodies in the ordinary space. However, clearly these laws are the same because the kinematical circumstances with respect to the local moving QS are closely the same in all these worlds.

\section{Conclusion}

This work shows that, in the light of recent new experimental observations and within the new theoretical scenario of the Higgs mechanism, Einstein's interpretation of the null results of the Michelson light anisotropy experiments definitely is wrong and that fundamental assumptions in the theory of relativity (TR) are scientifically incorrect. However, despite this, many of the conclusions of the TR closely match experimental observations. In fact this is illusory because all the experimental tests of the TR were made within earth based laboratories and not in free space. Due to the spacedynamic nature of the gravitational fields, evidenced by several recent clear-cut experimental observations, earth based laboratories are very closely resting with respect to the Higgs quantum space, ruling the inertial motion of matter and the propagation of light. In this way earth based laboratories accomplish very closely the kinematical circumstances that Einstein postulated for the resting observer in his thought experiments to give support to the TR. From the viewpoint of the present theory of spacedynamics, all the so called relativistic effects can be understood in terms of scientifically sound assumptions, as simple and genuine physical effects, due to motions with respect to the Higgs quantum space, taking into account that this quantum space is circulating round earth, round the sun and round each matter body throughout the universe according to a Keplerian velocity field, consistently with the local main astronomical motions, thereby creating the respective gravitational fields. Concomitantly, in the corresponding expressions for time dilation, for the relativistic mass and for the relativistic energy, found by Einstein, the relative velocity must be replaced by the velocity with respect to the Higgs quantum space.

\section{References}

[1] Lorentz, H.A., Einstein, A., Minkowski, H. and Weyl, H. (1923) The Principle of Relativity. Dover Publications, New York.

[2] Ives, H.E. and Stilwell, G.R. (1938) Journal of the Optical Society of America, 28, 215-219. http://dx.doi.org/10.1364/JOSA.28.000215

[3] Eisberg, R. and Resnick, R. (1974) Quantum Physics. John Willey and Sons Inc., Boston.

[4] Schaf, J. (2012) Journal of Modern Physics, 3, 714-749. http://dx.doi.org/10.4236/jmp.2012.38097

[5] Schaf, J. (2014) Journal of Modern Physics, 5, 407-448. http://dx.doi.org/10.4236/jmp.2014.56053

[6] Schaf, J. (2014) Recent Progress in Space Technology, 4, 44-66.

[7] Dixon, L. (1996) From Superconductors to Supercolliders. www.slac.stanford.edu/pubs/beamline/26/1/26-1-dixon.pdf

[8] Higgs, P.W. (1964) Physical Review Letters, 13, 508. http://dx.doi.org/10.1103/PhysRevLett.13.508

[9] Englert, F. and Brout, R. (1964) Physical Review Letters, 13, 321-323. http://dx.doi.org/10.1103/PhysRevLett.13.321

[10] Kibble, T.W.B. (2009) Scholarpedia, 4, 8741. http://dx.doi.org/10.4249/scholarpedia.8741

[11] Hatch, R.R. (2007) Physics Essays, 20, 83-100. http://dx.doi.org/10.4006/1.3073811

[12] Miller, D.C. (1933) Review of Modern Physics, 5, 203-242. http://dx.doi.org/10.1103/RevModPhys.5.203

[13] Hatch, R.R. (2004) GPS Solutions, 8, 67-73. http://dx.doi.org/10.1007/s10291-004-0092-8

[14] Hatch, R.R. (2004) Foundations of Physics, 34, 1725-1739. http://dx.doi.org/10.1007/s10701-004-1313-2

[15] Pound, R.V. and Snider, J.L. (1965) Physical Review B, 140, B788-B893. http://dx.doi.org/10.1103/PhysRev.140.B788

[16] Brault, J.W. (1963) Bulletin of the American Physical Society, 8, 28.

[17] Ashby, N. (2012) Private Communication.

[18] Bailey, H., Borer, K., Combley, F., Drumm, H. and Krienen, F. (1977) Nature, 268, 301-305. 
Scientific Research Publishing (SCIRP) is one of the largest Open Access journal publishers. It is currently publishing more than 200 open access, online, peer-reviewed journals covering a wide range of academic disciplines. SCIRP serves the worldwide academic communities and contributes to the progress and application of science with its publication.

Other selected journals from SCIRP are listed as below. Submit your manuscript to us via either submit@scirp.org or Online Submission Portal.
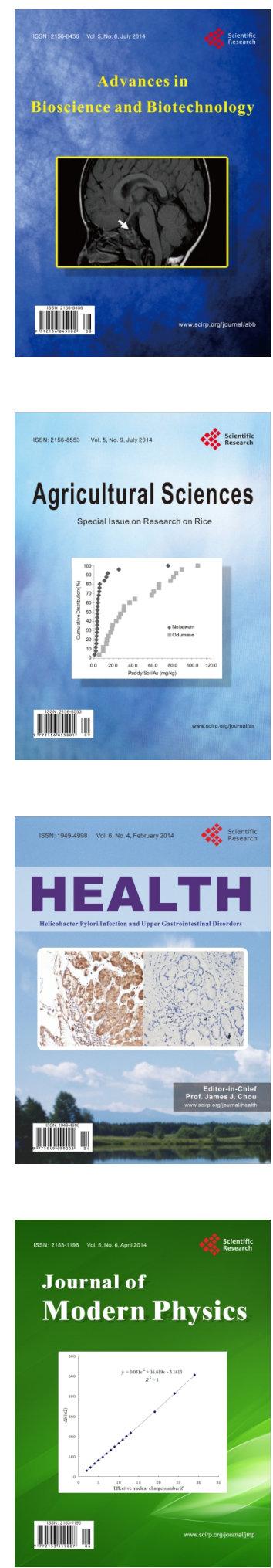
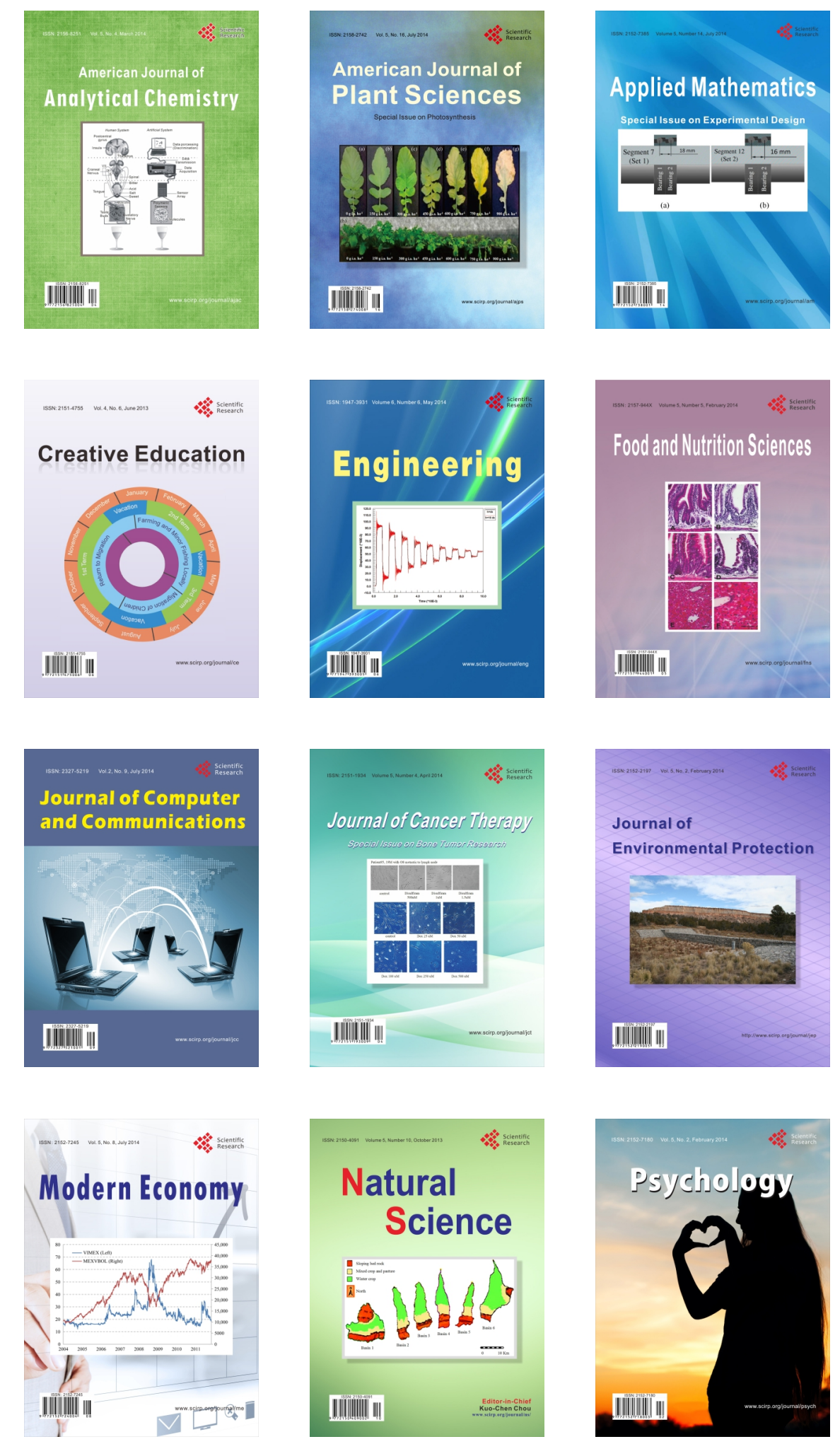